\title{
Calculation of the Dispersion of Specific Refractive Index Increments
}

Values of the specific refractive index increment, $\nu \equiv d n / d c$, are needed for the determination of molecular weights by light scattering. In particular for the study of copolymers it is often important to mask one of the monomers, i.e., to make $\nu$ of that monomer (nearly) zero by choosing an appropriate solvent and wavelength: The finer adjustments of $\nu$ can be achieved by the selection of the wavelength. It would therefore be convenient if dispersions in $\nu$ could be calculated in a simple way. From the LorenzLorentz equation one may derive ${ }^{1}$ for $c \rightarrow 0$

$$
\nu \equiv d n / d c=\frac{\left(n_{1}^{2}+2\right)^{2}}{6 n_{1} \rho_{2}}\left(\frac{\rho_{2} R_{2}}{M_{2}}-\frac{\rho_{1} R_{1}}{M_{1}}\right)
$$

Here $n$ is the refractive index of the solution; $c$ is the polymer concentration; $n_{1}$ is the refractive index of the solvent; $\rho_{2}$ and $\rho_{1}$ are the densities of polymer and solvent; $M_{2}$ and $R_{2}$ are the molecular weights and the molar refractions of the repeating units of the polymer; and $M_{1}$ and $R_{1}$ are those of the solvent. Although it is well-known that eq. (1) gives rather poor results for the absolute values of $\nu$, it might be expected to give much better accuracy for the dispersion in $\nu$. In Table $I$, calculated and experimental values of $\Delta \equiv \nu(436 \mathrm{~nm})-\nu(546 \mathrm{~nm})$ are compared. Only $\nu$ 's specified to four significant figures were used as tabulated by Huglin. ${ }^{2}$ Values of $R$ were calculated from Eisenlohr's bond refractions; ${ }^{3} n_{1}$ and $\rho_{1}$ were obtained from tabulations of Huglin ${ }^{4}$ at $20^{\circ} \mathrm{C}$, and $\rho_{2}$ from tabulations of Van Krevelen. ${ }^{5}$ The molar refractions of the phenyl group were obtained from averaged values of the molar refractions of monosubstituted benzene derivatives minus the bond refractions of the substituents $\left(\mathrm{R}\left(\mathrm{C}_{6} \mathrm{H}_{5}\right)=25.20,25.96\right.$, 26.44 , respectively, at $656.3,486.1,434 \mathrm{~nm}$ ). The molar refractions of the monosubstituted benzene derivatives were calculated from densities and refractive indexes, using the Lorenz-Lorentz equation.

Apparently the accuracy of the calculated $\Delta$ is $0.0020 \mathrm{ml} / \mathrm{g}$ or better. The following additional comments can be made:

1. There is no correlation between the magnitude of $\Delta$ and of $\Delta$ (calc) $-\Delta$ (exp).

2. When neither polymer nor solvent contains benzene rings, $\Delta$ is several times smaller than when polymer or solvent contain benzene rings.

3. $\Delta$ is negative when only the solvent contains a benzene ring. The existence of negative values of $\Delta$ is not always recognized. ${ }^{6}$

TABLE I

Dispersion of Refractive Index Increments ${ }^{2}$

\begin{tabular}{llrr}
\hline \multicolumn{1}{c}{ Polymer } & \multicolumn{1}{c}{ Solvent } & \multicolumn{1}{c}{$\Delta_{\text {calo. }}$} & \multicolumn{1}{c}{$\Delta_{\text {calc. }}-\Delta_{\text {exp. }}$} \\
\hline Poly(ethyl acrylate) & butanone & 0.0014 & 0.0000 \\
Poly(propylene oxide) & isooctane & 0.0007 & 0.0007 \\
Poly(methyl methacrylate) & isoamyl acetate & 0.0020 & 0.0000 \\
Poly(n-butyl methacrylate) & acetone & 0.0014 & 0.0001 \\
Poly(propylene oxide) & benzene & -0.0100 & -0.0018 \\
Poly(propylene oxide) & chlorobenzene & -0.0100 & -0.0020 \\
Poly(methyl methacrylate) & benzene & -0.0056 & 0.0015 \\
Poly(methyl methacrylate) & toluene & -0.0052 & 0.0021 \\
Polystyrene & cyclohexane & 0.0105 & -0.0008 \\
Polystyrene & butanone & 0.0109 & -0.0017 \\
Poly( $\alpha$-methylstyrene) & carbon tetrachloride & 0.0086 & -0.0014 \\
Poly( $\alpha$-methylstyrene) & cyclohexane & 0.0098 & -0.0022 \\
Polystyrene & benzene & 0.0026 & -0.0006 \\
Polystyrene & toluene & 0.0029 & -0.0005 \\
\hline
\end{tabular}

(C) 1975 by John Wiley \& Sons, Inc. 


\section{References}

1. J. W. Lorimer, Polymer, 13, 46 (1972).

2. M. B. Huglin in Light Scattering from Polymer Solutions, M. B. Huglin, Ed., Academic Press, London, 1972, Chap. 6.

3. F. Eisenlohr, Z. Phys. Chem., 75, 585 (1910); 79, 129 (1912).

4. Ref. 2, p. 29.

5. D. W. van Krevelen in Properties of Polymers, Elsevier, Amsterdam, 1972, Appendix 2.

6. Ref. 2, p. 195.

\section{J. LAVEAN}

M. W. J. van DEN EsKer

A. VRIJ

Van't Hoff Laboratorium

voor Fysische en Colloidchemie .

der Rijksuniversiteit

Padualaan 8

Utrecht, The Netherlands

Received April 15, 1974

Revised August 27, 1974 